Tropical Journal of Pharmaceutical Research April 2019; 18 (4): 877-888

ISSN: $1596-5996$ (print); 1596-9827 (electronic)

(c) Pharmacotherapy Group, Faculty of Pharmacy, University of Benin, Benin City, 300001 Nigeria.

\title{
Efficacy of calcium sodium phosphosilicate containing dentifrice in reducing dentin hypersensitivity compared to other dentifrices with dentin tubule occluding molecules: A systematic review
}

\author{
Sunil Kumar Vaddamanu1, Saad M AlQahtani ${ }^{2}$, Ravi Kadur Sundarraj ${ }^{3 \star}$, \\ Raghavendra Reddy Nagate ${ }^{4}$, Vijay Apparaju ${ }^{5}$ \\ ${ }^{1}$ Department of Dental Technology, ${ }^{2}$ Department of Periodontics and Community Dental Sciences, College of \\ Dentistry, ${ }^{3}$ Department of Pediatric Dentistry and Orthodontic Sciences, College of Dentistry, ${ }^{4}$ Department of Periodontics and \\ Community Dental Sciences, College of Dentistry, King Khalid University, Abha, Kingdom of Saudi Arabia, ${ }^{5}$ Department of \\ Periodontics, Dr Vijay's Multispeciality Dental Care, Bangalore, India
}

*For correspondence: Email: sundarraj@kku.edu.sa

Sent for review: 20 November 2018

Revised accepted: 15 March 2019

\begin{abstract}
Purpose: To assess the effectiveness of calcium sodium phosphosilicate in reducing dentin hypersensitivity compared to other dentin tubule occluding molecules.

Methods: A structured research question was formulated, and an electronic search of available literature was carried out via PubMed, Google Scholar, and Scopus. A hand search as well as a gray literature search were also carried out. The search produced a total of 67 articles. Of these, only eight articles were eligible to be included in our review. Risk of bias and study quality were checked using Cochrane tool. The review was registered in The International Prospective Register of Systematic Reviews (PROSPERO) under registration number CRD42018096200.

Results: The results show a reduction in dentin hypersensitivity with calcium sodium phosphosilicate compared to many other molecules. However, nanohydroxyapatite showed a better desensitizing effect compared to Novamin.

Conclusion: According to the available evidence, $5 \%$ calcium sodium phosphosilicate containing toothpaste is more effective reducing dentin hypersensitivity compared to many other dentinal tubule occluding molecules.
\end{abstract}

Keywords: Dentin hypersensitivity, novamin, calcium sodium phosphosilicate, potassium nitrate

\begin{abstract}
This is an Open Access article that uses a fund-ing model which does not charge readers or their institutions for access and distributed under the terms of the Creative Commons Attribution License (http://creativecommons.org/licenses/by/4.0) and the Budapest Open Access Initiative (http://www.budapestopenaccessinitiative.org/read), which permit unrestricted use, distribution, and reproduction in any medium, provided the original work is properly credited.

Tropical Journal of Pharmaceutical Research is indexed by Science Citation Index (SciSearch), Scopus, International Pharmaceutical Abstract, Chemical Abstracts, Embase, Index Copernicus, EBSCO, African Index Medicus, JournalSeek, Journal Citation Reports/Science Edition, Directory of Open Access Journals (DOAJ), African Journal Online, Bioline International, Open-J-Gate and Pharmacy Abstracts
\end{abstract}

\section{INTRODUCTION}

Dentin hypersensitivity $(\mathrm{DH})$ is usually defined as acute sharp lingering pain associated with exposure of dentinal tubules to thermal, evaporative, tactile, or chemical stimuli which can't be attributed to any other dental pathology or anomaly [1].

Several etiologies are associated with $\mathrm{DH}$. 
Gingival recession is the most frequent etiology of $\mathrm{DH}$, followed by attrition and erosions. Dentinal tubule exposure due to dental caries and attrition usually occurs in children and young adults. Meanwhile, gingival recession due to periodontal disease and following periodontal treatment is more frequent in older patients. In addition, excessive occlusal force, premature occlusion, erosion, or abrasion due to over jealous tooth brushing may cause enamel loss and subsequently $\mathrm{DH}$ [2]. Dentin hypersensitivity affects 3 to $98 \%$ of general population and, on average, $15 \%$ of adult population [3].Although $\mathrm{DH}$ affects various age groups, its peak prevalence occurs at $30-40$ years age group. In addition, females are more affected by $\mathrm{DH}$ than males [3].Dentin hypersensitivity is one of the main problems for which patients seek dental treatment [2].

Several theories were proposed to explain $\mathrm{DH}$ includingdirect innervation theory, odontoblast repair theory, and hydrodynamic/fluid moment theory. Of these, hydrodynamic theory is the most widely accepted [1,2]. According to hydrodynamic theory, any fluid moment in dentinal tubules may stimulate nerve fibers. Hence, targeting dentinal tubule occlusion or blocking nerve conduction may reduce $\mathrm{DH}$ [4]. Dentifrices with dentinal tubule occluding moleculesor potassium or sodium salts (thatdecrease nerve transmission), laser therapy, and iontophoresis are some of the proposed $\mathrm{DH}$ treatment methods [5].Potassium salts act as nerve-numbing agentsby increasing potassium ion concentration in extracellular dentinal fluids [6].Nevertheless, according to few clinical studies, this effect of potassium salts (potassium nitrate)is transient [7].

Several clinical studies showed calcium sodium phosphosilicate (CSPS) to havesuperior desensitizing effectcompared to potassium nitrate [4,6,8-10]. Calcium sodium phosphosilicate is a bioactive glass material that reacts with saliva to form hydroxyapatite-like crystals on dentinal surface. This newly-formed mineralized layer dentinhas the same mineral content as bone, enamel, and dentin.Furthermore, it acts as a barrier against oral fluids preventing further $\mathrm{DH}[11]$.

Several systematic reviews were conducted on Novamin (a dentifrice containing calcium sodium phosphosilicate). One studyreviewedclinical trials comparing CSPS with placebo [12]. Another review included studies comparing CSPS to other desensitizing dentifrices [13]. However, none of previous systematic reviews did review studies comparing CSPS to other dentinal tubule occluding molecules. Therefore, in this study we review studies comparing CSPS to other dentinal tubule occluding molecules.

\section{METHODS}

\section{Protocol and registration}

This study was conducted according to PRISMA guidelines (Preferred Reporting Items for Systematic review and Meta-Analysis)[14]. Study protocol was registered in PROSPERO (International prospective registration of systematic reviews) under registration no. CRD42018096200.

\section{Research question}

We systematically reviewed randomized clinical trials (RCTs) to investigate our research question. Our formulated research question was "What is the immediate and long-term efficacy reducing dentin hypersensitivity of dentifrice containing calcium sodium phosphosilicate (Novamin) compared to dentifrices containing other dentin tubule occluding molecules in patients with dentin hypersensitivity?".

\section{Search strategy}

PubMed (MEDLINE), Scopus, and Google scholar were searched for studies published till July 2018 without any language restrictions. Search was conducted in PubMed (MEDLINE) with Mesh terms and keywords. Search details were "Search ("NOVAMIN"[Title/Abstract]) OR "Calcium sodium phosphosilicate"[Title/Abstract]) OR "Bioactive glass"[Title/Abstract])) AND ("sensitive tooth"[Title/Abstract]) OR "dental hypersensitivity" [Title/Abstract]) OR "dentinal hypersensitivity" [Title/Abstract]) OR "dentinal sensitivity" [Title/Abstract]) OR "dentin sensitivity" [Title/Abstract]) OR "dental sensitivity" [Title/ Abstract]) OR "tooth hypersensitivity" [Title/Abstract]) OR "sensitivity"[Title/Abstract]) OR "Hypersensitivity"[Title/Abstract]) Filters: Randomized Controlled Trial; Publication date to 2018/07/31AND "humans" [MeSH Terms])".Filter options were utilized to further limit search results. Searching was conducted by two separate blinded researchers (VA and VSK).In addition, we searched OpenGray, ClinicalTrials.gov, WHO clinical trials registration platform, and Google Scholar for gray literature. A separate hand search was also conducted reviewing references of electronic search results. Inter-raters reliability was assessed by Cohen's kappa coefficient. 


\section{Inclusion and exclusion criteria}

Criteria for study inclusion in review are mentioned in Table 1.

Table 1: Inclusion criteria

\begin{tabular}{ll}
\hline P: Participants & Patients with $\mathrm{DH}$. \\
I: Intervention & Application of Novamin \\
& molecule in any form, any \\
& concentration, and at any \\
& frequency.
\end{tabular}

C: Comparison

Comparing with dentifrice containing other dentin tubule occluding molecule or treatments which occlude dentinal tubules (laser therapy, iontophoresis, dental varnishes, etc)

O: Outcome

S: Studies

\begin{abstract}
Primary outcome: Reduction of $\mathrm{DH}$, evaluated for at least four weeks.

Secondary outcome: Any uneventful events like allergic reactions associated.
\end{abstract}

Randomized clinical trials (RCTs)

- Observational studies, animal studies, In-vitro studies, letters to editors, and reviews all were excluded from review. In addition, we excluded studies with patients having any systemic disease, who are already undergoing any treatments or undergone any procedures for $\mathrm{DH}$, who are using analgesics, with tooth fractures, or with post-restoration $\mathrm{DH}$. Studies with improper methodologies such as, Improper or no measuring tool for $\mathrm{DH}$, inappropriate or unpublished results, or no patient follow-up were also excluded. Finally, studies where low-power laser therapy was used were excluded as well, as low-power laser therapy would affect nerve transmission rather than dentin tubule occlusion.

Search results were first screened by titles and abstracts by two blinded reviewers (NRR and VSK). Any disagreements were resolved by discussion, if required, with a third researcher to reach a consensus. Authors of the reviewed publications were contacted by a fourth researcher when ever needed. Duplicate search results were eliminated. Remaining studies were subjected to full-text evaluation.

\section{Data extraction}

Data extraction was done by two independent researchers. Any disagreementswere resolved by discussionwith a third researchertoreach a consensus. For each study, data were extracted regarding year of publication, author names, study location, number of participants, age range and mean age of participants, study groups, interventions used, type of stimulus used, follow up intervals and maximum follow-up period, and primary and secondary outcomes of interest.

\section{Assessment of study quality and bias}

Risk of bias was evaluated by two separate reviewers according to instructions of Cochran handbook of systematic reviews of intervention.. Studies were assessed for randomization, allocation of participants, blinding of participants and outcome, incomplete outcome data, and selective reporting. Overall good and fair quality studies were included for review, while poor quality studies were excluded from review. Any disagreement between the two reviewers was clarified by a discussion between both of them or with a third author to reach a consensus. Reviewers checked acknowledgments in studies and author's disclosure forms for conflicts of interests based on Friedman and Richter criteria. For missing data and unpublished information, another researcher contacted corresponding authors when needed. I $^{2}$ analysis was used to assess study heterogeneity. Due to significant variations in studies protocols and follow-up periods, a meta-analysis wasn't carried out.

\section{RESULTS}

\section{Study selection}

Our initial search produced a total of 67 results. Twenty-one duplicate records and seven unrelated articles (screened out by reviewing titles and abstracts) were excluded. Finally, 14 articles undergone full-text evaluation. After eliminating seven ineligible and poor quality articles, eight articles [4,6,8-10,15-17] were included in this review. Figure 1 shows a flowchart of study selection for review.

\section{Characteristics of included studies}

A detailed description of the eight included studies is mentioned in Table 2. Quality assessment summary and quality of individual studies are stated in Table 3.

\section{Study outcomes}

In all included studies, Novamin was used at a concentration of $5 \% \quad[4,6,8-10,15,16]$. Five $[4,6,8-10]$ out of eight studies showed Novamin 


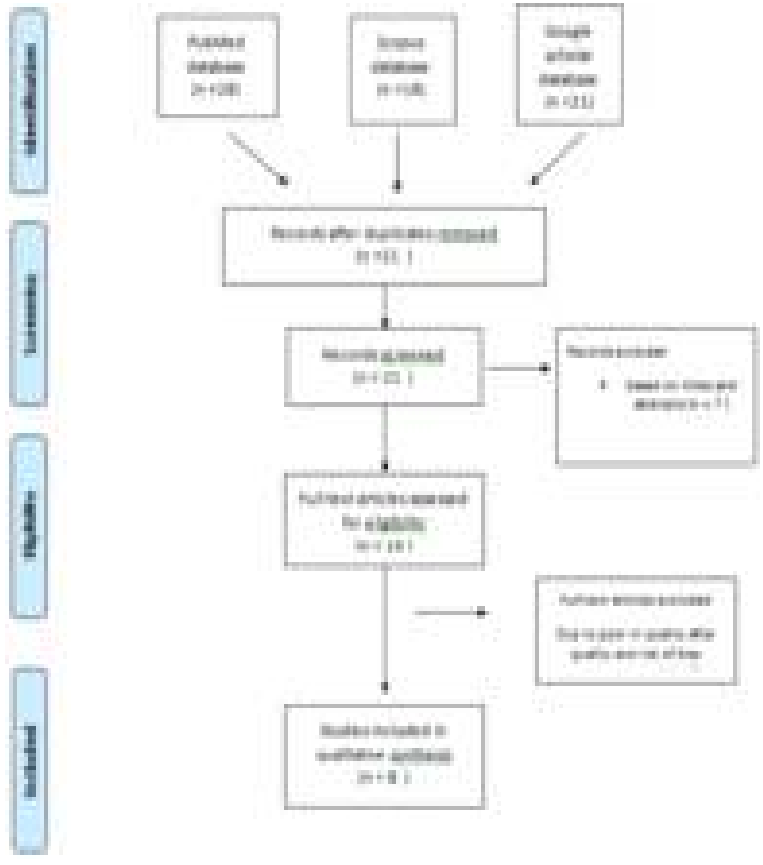

Figure 1: Flowchart showing study design according to PRISMA 2009 guidelines

to be superior to comparison molecule, while, three studies [15-17] showed comparison molecules(arginine, nanohydroxyapatite, and fluoro calcium phosphosilicate) to be superior to Novamin. Out of eight included studies, five studies compared Novamin with $5 \%$ potassium nitrate $[4,6,8-10]$. These five studies all showed Novamin to be superior to potassium nitrate regarding reduction in $\mathrm{DH}$. One study with a maximum follow-up period of 12 weeks showed a mean visual analog scale (VAS) score of 8.06 to 3.37 with Novamin, which was significantly different from potassium nitrate (baseline VAS = 7.20 and at 12 weeks $=5.00$ ) [10]. Meanwhile, one out of eight included studies compared Novamin to $8 \%$ arginine [16]. Surprisingly, this study showed arginine to be superior to Novamin regarding mean reduction in $\mathrm{DH}$. Novamin was compared to $3.85 \%$ amine fluoride in two of the included studies $[4,10]$.

In both studies, Novamin showed superior effects compared to amine fluoride after six weeks, and even after twelve weeks in one of the two studies [10]. Two studies compared Novamin with nano hydroxyapatite particles. In both studies, nanohydroxyapatite showed superior results compared to Novamin at follow-up after four weeks $[15,16]$. One study compared Novamin to $5 \%$ fluoro calcium phosphosilicate and showed fluoro calcium phosphosilicate to be superior to Novamin even after eight-week follow-up. Notably, none of the included studies reported anyadverse effects associated with the use of Novamin.

\section{Risk of bias and quality assessment}

After assessing the risk of bias in different aspects using Cochrane collaboration tool, final quality of studies were evaluated. Seven studies were excluded because of poor quality and high risk associated with their inclusion in review.

\section{DISCUSSION}

The current systematic review was conducted to assess evidence regarding effect of Novamin on dentin hypersensitivity (DH) compared to other dentinal tubule occluding molecules. We also aimed to assess immediate and long-term adverse effects of Novamin. In this systematic review, we aimed to only consider randomized controlled clinical trials for inclusion. Studies in which Novamin was compared with placebo were excluded. Only RCTs of at least one dentinal tubule occluding molecule in addition to Novamin were included.

Due to the similarity to bone mineral, calcium sodium phosphosilicate was proposed in the late nineteenth century as a regenerating materia [24]. Later, this molecule was introduced to the field of oral care for repairing damaged dentinal surfaces. Novamin is nothing but a calcium sodium phosphosilicate molecule which can occlude dentinal tubules by forming a mineralized layer on the exposed dentinal tubules. The newly-formed layer of Novamin is proposed to be resistant to $\mathrm{pH}$ fluctuations of saliva, and therefore resistant to dislodgment off dentinal surface [24]. A recent systematic review of studies comparing Novamin with placebo concluded that Novamin is effective in reducing DH compared to a negative control [12]. In addition, Novamin was compared to various dentinal tubule occluding molecules.

West et al reviewed effectiveness of several professional and self-administered desensitizing agents, and concluded that Novamin and strontium chloride were more effective compared to other molecules [5]. Another summary review [1] suggested superiority of Novamin over comparison, but surprisingly showed strontium chloride to have no advantage over placebo which is contradicting results of a previous systematic review [5]. 
Table 2: Characteristics of RCTs included in review

\begin{tabular}{|c|c|c|c|c|c|c|c|c|c|c|}
\hline S.NO & $\begin{array}{l}\text { Year of } \\
\text { publication }\end{array}$ & Author name & $\begin{array}{l}\text { Place of } \\
\text { study } \\
\text { conducted }\end{array}$ & $\begin{array}{l}\text { Number } \\
\text { of } \\
\text { participan } \\
\text { ts }\end{array}$ & $\begin{array}{l}\text { Age range } \\
\text { and mean } \\
\text { age }\end{array}$ & $\begin{array}{l}\text { Groups and } \\
\text { Active ingredients } \\
\text { used in } \\
\text { dentifrices }\end{array}$ & $\begin{array}{l}\text { Concentrati } \\
\text { on used }\end{array}$ & $\begin{array}{l}\text { Type of } \\
\text { stimulu } \\
\text { s used }\end{array}$ & $\begin{array}{l}\text { Scale } \\
\text { used to } \\
\text { measure } \\
\text { DH }\end{array}$ & $\begin{array}{l}\text { Follow up } \\
\text { intervals } \\
\text { and } \\
\text { maximum } \\
\text { follow-up } \\
\text { period }\end{array}$ \\
\hline \multirow[t]{3}{*}{1.} & \multirow[t]{3}{*}{2010} & \multirow[t]{3}{*}{$\begin{array}{l}\text { Narongdej et } \\
\text { al[18] }\end{array}$} & \multirow[t]{3}{*}{ Thailand } & \multirow[t]{3}{*}{60} & \multirow[t]{3}{*}{$\begin{array}{l}26-70 \\
\text { years } \\
\text { Mean age } \\
44.8 \text { years }\end{array}$} & $\begin{array}{l}\text { G1: Novamin } \\
\text { powder+ Novamin } \\
\text { containing tooth } \\
\text { paste }\end{array}$ & $\begin{array}{l}100 \% . \text { and } \\
7.5 \%, \\
\text { respectively }\end{array}$ & \multirow[t]{3}{*}{$\begin{array}{l}\text { Thermal } \\
\text { and } \\
\text { Tactile }\end{array}$} & \multirow[t]{3}{*}{$\begin{array}{l}\text { VAS* scale }^{*} \\
(0-10)\end{array}$} & \multirow{3}{*}{$\begin{array}{l}\text { Before, } \\
\text { baseline, } \\
\text { one week, } \\
\text { two } \\
\text { weeks, } \\
\text { and four } \\
\text { weeks }\end{array}$} \\
\hline & & & & & & $\begin{array}{l}\text { G2: Tooth paste } \\
\text { containing } \\
\text { Novamin only and } \\
\text { placebo powder. }\end{array}$ & $7.5 \%$ & & & \\
\hline & & & & & & $\begin{array}{l}\text { G3: Tooth paste } \\
\text { containing } \\
\text { Potassium } \\
\text { nitrate and sodium } \\
\text { fluoride. }\end{array}$ & $\begin{array}{l}5 \% \text { and } \\
0.221 \% \\
\text { respectively. }\end{array}$ & & & \\
\hline \multirow[t]{3}{*}{2.} & \multirow[t]{3}{*}{2010} & \multirow[t]{3}{*}{$\begin{array}{l}\text { Pradeep and } \\
\text { Sharma[6] }\end{array}$} & \multirow[t]{3}{*}{ India } & \multirow[t]{3}{*}{110} & \multirow{3}{*}{$\begin{array}{l}20-60 \\
\text { years } \\
\text { Mean } \\
\text { age40 } \\
\text { years }\end{array}$} & G1: Novamin & $5 \%$ & \multirow[t]{3}{*}{$\begin{array}{l}\text { Evaporati } \\
\text { ve and } \\
\text { thermal }\end{array}$} & \multirow[t]{3}{*}{$\begin{array}{l}\text { VAS scale } \\
(0-10)\end{array}$} & \multirow{3}{*}{$\begin{array}{l}\text { Baseline, } \\
2 \text { weeks, } \\
\text { and } 6 \\
\text { weeks }\end{array}$} \\
\hline & & & & & & $\begin{array}{l}\text { G2: Potassium } \\
\text { nitrate }\end{array}$ & $5 \%$ & & & \\
\hline & & & & & & $\begin{array}{l}\text { G3: Tooth paste } \\
\text { without any } \\
\text { desensitizing } \\
\text { agents }\end{array}$ & $\mathrm{Nil}$ & & & \\
\hline
\end{tabular}


Table 2: Characteristics of RCTs included in review (continued)

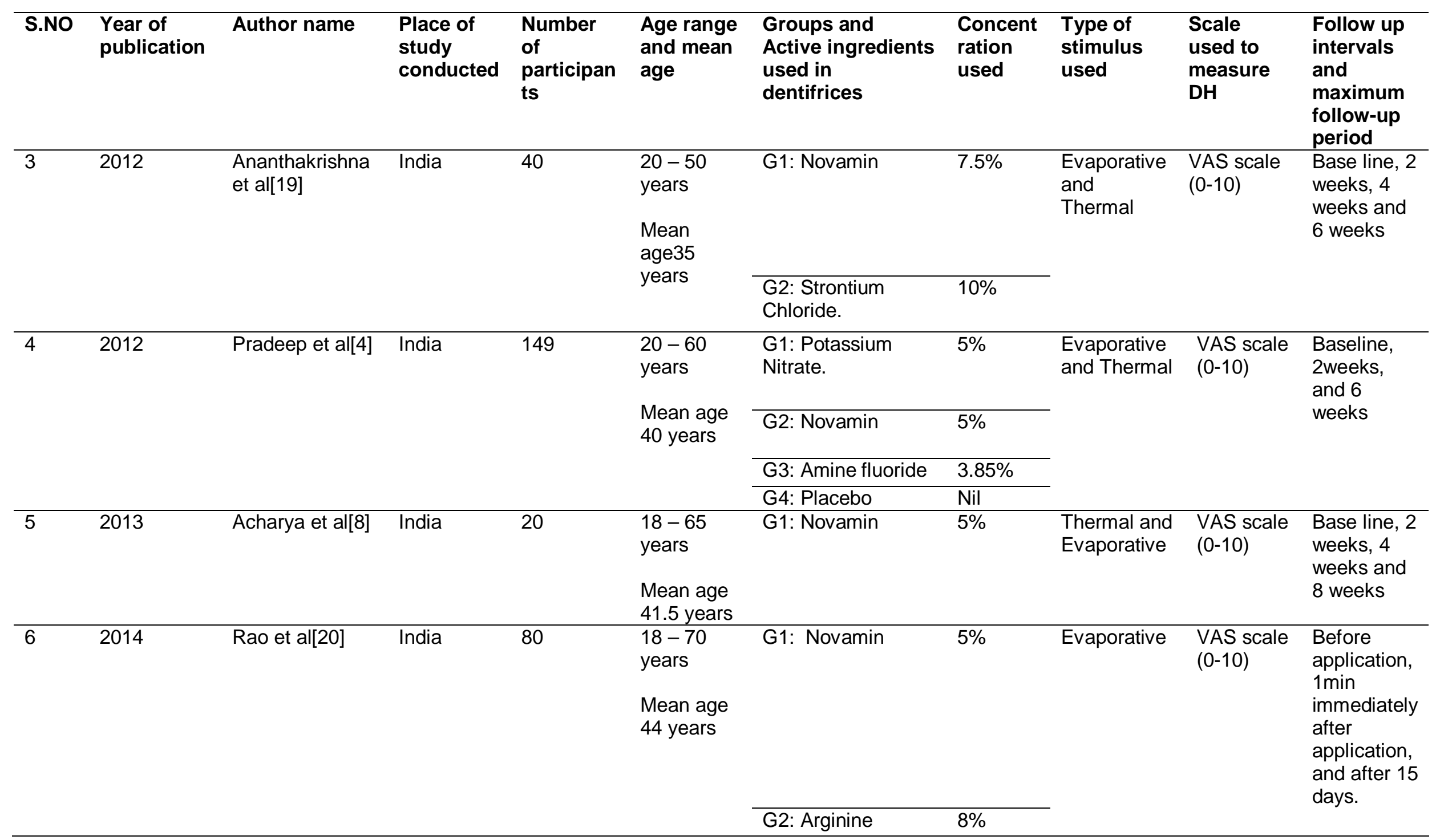


Table 2: Characteristics of RCTs included in review (continued)

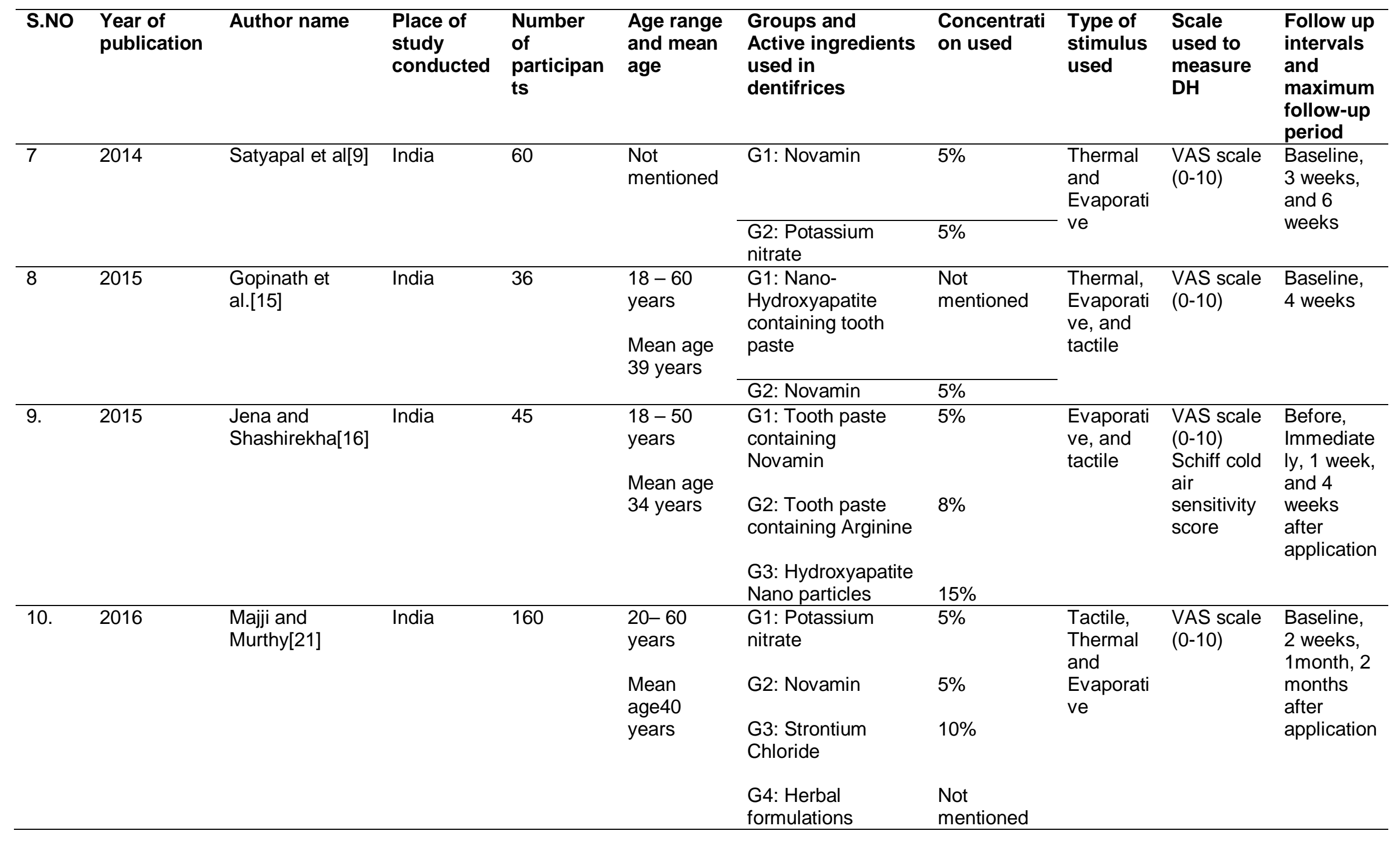


Table 2: Characteristics of RCTs included in review (continued)

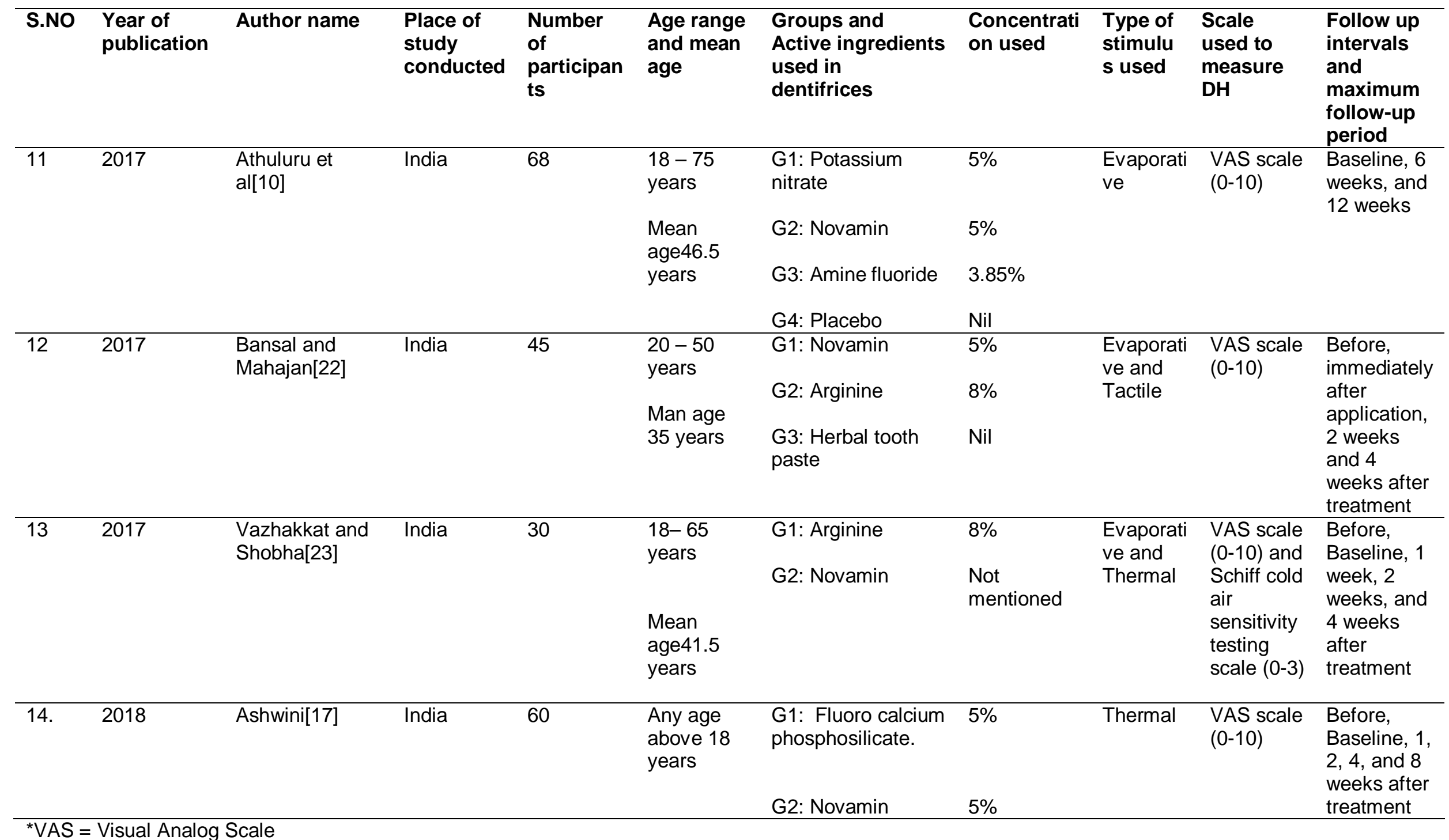


Table 3: Summary of risk of bias assessment

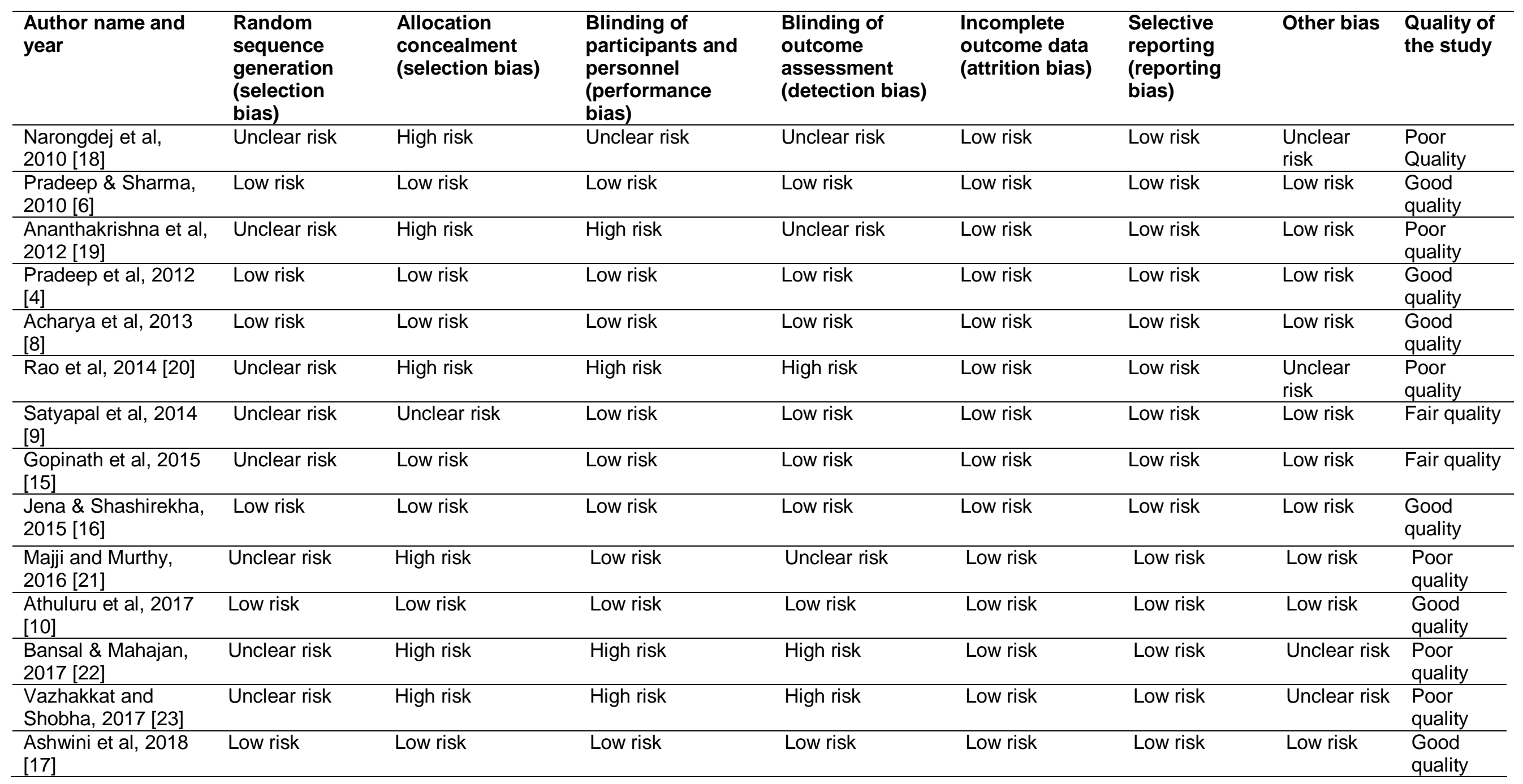


Another systematic review by Bae et al [13] also supported the efficacy of Novamin along with other dentinal tubule occluding molecules, and highlighted the non-superiority of strontium chloride over placebo. These results are also similar to Levenson's review results [1].

Recent clinical trials comparing Novamin with nanohydroxyapatite particles $[15,16]$, highlighted the superiority of nanohydroxyapatite particles over Novamin. Cold and tactile tests at four-week follow-up showed a superior reduction in mean VAS scores with nanohydroxyapatite particles, but evaporative stimulus showed Novamin to be superior in reducing baseline mean VAS score in a study by Gopinath et al [15]. Dentin tubule occlusion capacity of Novamin was also tested in in-vitro studies using scanning electron microscopy [25]. However, this microscopy study revealed lower tubular occlusion capacity and resistance after acid challenge of Novamin when compared to arginine-calcium carbonate and propolis extract. Another in-vitro study confirmed formation of hydroxyapatite like crystals when CSPS is mixed with saliva on dentin slabs [26].

Dentin hypersensitivity usually occurs due to exposure of dentinal surface to the oral environment due to loss of gingiva, decay, or after periodontal surgery. Dentin hypersensitivity following periodontal surgery might be due to the inadvertent removal of cementum during root planing procedure and apical shift of marginal gingiva after the procedure [2]. Dentin hypersensitivity occurring due to decay or gingival recession differs from $\mathrm{DH}$ occurring after periodontal surgery. Dentin hypersensitivity occurring after periodontal surgery usually peaks immediately after surgery and improves spontaneously after a few days. Hence, treatment of $\mathrm{DH}$ occurring after periodontal surgical procedure could be postponed intentionally [2].

Although many clinical trials didn't use CSPS at concentrations higher than $5 \%$, various CSPS formulations are available in market with concentrations from 2.5 to $15 \%$. Concentrations ofprofessional-administered CSPS formulations are generally higher compared to home-use or self-administered ones [5].

Different positive controls were compared to CSPS. Potassium nitrate, arginine, amine fluoride, nanohydroxyapatite are the most commonly tested positive control molecules $[4,6,10,15,16]$. Another commonly used positive control molecule is fluoride salts [17]. Usage of fluoride-containing positive control is still controversial since a high concentration of fluorides would occlude dentinal tubules, while lower concentration reduces nerve conduction process [4]. Potassium nitrate was used as a positive control to assess efficacy of Novamin in many studies $[4,6,10,15]$ Potassium nitrate containing dentifrices also show dual mechanism of action as potassium salts would occlude dentinal tubules, while increased potassium ions would increase threshold of nerve conduction and finally block nerve conduction [18]. Although, United States FDA approved using potassium nitrate as a desensitizing agent and many clinical trials also support that, long-term desensitizing effects aren't evident.

\section{Study limitations}

This systematic review is mainly limited by the nature of included studies. Some of the included studies were sponsored by medical industries, therefore, raising potential conflicts of interests. While other trials had small sample size, short follow-up periods, or only one stimulus type used to check DH. In addition, all included fair-quality studies didn't provide information regarding randomization and allocation concealment which would potentially increase risk of bias.

\section{CONCLUSION}

According to results of this systematic review, 5 $\%$ CSPS containing tooth paste is expected to be more effective compared to many other dentinal tubule occluding molecules. However, evidence shows nanohydroxyapatite to be superior to CSPS regarding immediate and long-term desensitizing effects. Development of adverse effects with the usage of $5 \%$ CSPS-containing dentifrices wasn't reported in any of the included studies.

\section{DECLARATIONS}

\section{Acknowledgement}

We would like to acknowledge Dr Rajesh Vyas for his constant support and advice on literature search and data analysis.

\section{Conflict of Interest}

No conflict of interest associated with this work.

\section{Contribution of Authors}

The authors declare that this work was done by the authors named in this article and all liabilities pertaining to claims relating to the content of this article will be borne by them. 


\section{Open Access}

This is an Open Access article that uses a funding model which does not charge readers or their institutions for access and distributed under the terms of the Creative Commons Attribution License (http://creativecommons.org/licenses/by/ 4.0) and the Budapest Open Access Initiative (http://www.budapestopenaccessinitiative.org/rea d), which permit unrestricted use, distribution, and reproduction in any medium, provided the original work is properly credited.

\section{REFERENCES}

1. Levenson D. Beneficial effects seen with most desensitising toothpastes. Evid Based Dent 2016; 17(1): 10-11.

2. Dowell P, Addy M. Dentine hypersensitivity--a review. Aetiology, symptoms and theories of pain production. $J$ Clin Periodontol 1983; 10(4): 341-350.

3. Splieth $\mathrm{CH}$, Tachou A. Epidemiology of dentin hypersensitivity. Clin Oral Investig 2013; 17 Suppl 1: S38.

4. Pradeep AR, Agarwal E, Naik SB, Bajaj P, Kalra N. Comparison of efficacy of three commercially available dentifrices [corrected] on dentinal hypersensitivity: a randomized clinical trial. Aust Dent J 2012; 57(4): 429434.

5. West NX, Seong J, Davies M. Management of dentine hypersensitivity: efficacy of professionally and selfadministered agents. J Clin Periodontol 2015; 42 Suppl 16: S256-302.

6. Pradeep AR, Sharma A. Comparison of clinical efficacy of a dentifrice containing calcium sodium phosphosilicate to a dentifrice containing potassium nitrate and to a placebo on dentinal hypersensitivity: a randomized clinical trial. J Periodontol 2010; 81(8): 1167-1173.

7. Salian S, Thakur S, Kulkarni S, LaTorre G. A randomized controlled clinical study evaluating the efficacy of two desensitizing dentifrices. J Clin Dent 2010; 21(3): 8287.

8. Acharya A, Surve S, Thakur S. A clinical study of the effect of calcium sodium phosphosilicate on dentin hypersensitivity. J Clin Exp Dent 2013; e18-22.

9. Satyapal $T$, Mali $R$, Mali A, Patil V. Comparative evaluation of a dentifrice containing calcium sodium phosphosilicate to a dentifrice containing potassium nitrate for dentinal hypersensitivity: A clinical study. J Indian Soc Periodontol 2014; 18(5): 581-585.

10. Athuluru D, Reddy C, Sudhir KM, Kumar K, Gomasani S, Nagarakanti S. Evaluation and comparison of efficacy of three desensitizing dentifrices on dentinal hypersensitivity and salivary biochemical characteristics: A randomized controlled trial. Dent Res J (Isfahan). 2017; 14(2): 150-157.
11. Kurt S, Kırtıloglu T, Yılmaz NA, Ertas E, Orucoglu $H$. Evaluation of the effects of Er:YAG laser, Nd:YAG laser, and two different desensitizers on dentin permeability: in vitro study. Lasers Med Sci. 2018; 33(9): 1883-1890.

12. Zhu M, Li J, Chen B, Mei L, Yao L, Tian J. The Effect of Calcium Sodium Phosphosilicate on Dentin Hypersensitivity: A Systematic Review and MetaAnalysis. PLoS One 2015; 10(11): e0140176.

13. Bae J-H, Kim Y-K, Myung S-K. Desensitizing toothpaste versus placebo for dentin hypersensitivity: a systematic review and meta-analysis. J Clin Periodontol 2015; 42(2): 131-141.

14. Moher D, Altman DG, Liberati A, Tetzlaff J. PRISMA statement. Epidemiology 2011; 22(1): 128.

15. Gopinath NM, John J, Nagappan N, Prabhu S, Kumar ES. Evaluation of Dentifrice Containing Nanohydroxyapatite for Dentinal Hypersensitivity: A Randomized Controlled Trial. J Int Oral Heal 2015; 7(8): 118-122.

16. Jena A, Shashirekha G. Comparison of efficacy of three different desensitizing agents for in-office relief of dentin hypersensitivity: A 4 weeks clinical study. J Conserv Dent 2015; 18(5): 389-393.

17. Ashwini S, Swatika K, Kamala DN. Comparative Evaluation of Desensitizing Efficacy of Dentifrice Containing 5\% Fluoro Calcium Phosphosilicate versus 5\% Calcium Sodium Phosphosilicate: A Randomized Controlled Clinical Trial. Contemp Clin Dent 2018; 9(3): 330-336.

18. Narongdej $T$, Sakoolnamarka $R$, Boonroung $T$. The effectiveness of a calcium sodium phosphosilicate desensitizer in reducing cervical dentin hypersensitivity: a pilot study. J Am Dent Assoc 2010; 141(8): 995-999.

19. Ananthakrishna S, Raghu TN, Koshy S, Kumar $N$. Clinical evaluation of the efficacy of bioactive glass and strontium chloride for treatment of dentinal hypersensitivity. J Interdiscip Dent 2012; 2(2): 92-97.

20. Rao A, Mitra D, Prabhakar AK, Soni S, Ahmed S, Arya S. The reduction efficacy of dentinal hypersensitivity by two commercially available desensitizing toothpastes: Vantej and Colgate Pro- Argin. Saudi Endod J 2014; 4(1): 712.

21. Maiji $P$, Murthy KRV. Clinical efficacy of four interventions in the reduction of dentinal hypersensitivity: A 2-month study. Indian J Dent Res 2016; 27(5): 477-482.

22. Bansal D, Mahajan M. Comparative Evaluation of Effectiveness of Three Desensitizing Tooth Pastes for Relief in the Dentinal Hypersensitivity. Contemp Clin Dent 2017; 8(2): 195-199.

23. Vazhakkat PR, Shobha K. Comparative Evaluation of the Effect of Two Desensitizing Dentifrices containing Novamin Technology and Pro-Argin Technology following Scaling and Root Planing.. J Heal Sci Res 2017; 8(1): 7-14.

24. Jones JR. Reprint of: Review of bioactive glass: From Hench to hybrids. Acta Biomater 2015; 23 Suppl: S5382. 
25. Chen CL, Parolia A, Pau A, Celerino de Moraes Porto IC. Comparative evaluation of the effectiveness of desensitizing agents in dentine tubule occlusion using scanning electron microscopy. Aust Dent $J$ 2015; 60(1): 65-72.
26. Earl JS, Leary RK, Muller KH, Langford RM, Greenspan DC. Physical and chemical characterization of dentin surface following treatment with NovaMin technology. J Clin Dent 2011; 22(3): 62-67. 